\title{
A Rare Arthrogryposis Syndrome with Multiple Anomalies Diagnosed by Whole Exome Sequencing
}

\author{
Audrey Rae Norby, Caitlin Madden Clifford*, Deborah Rose Berman \\ Department of Maternal Fetal Medicine, Michigan Medicine, Ann Arbor, USA \\ Email address: \\ audnorby@med.umich.edu (A. R. Norby), cmcliff@med.umich.edu (C. M. Clifford), debster@med.umich.edu (D. R. Berman) \\ ${ }^{*}$ Corresponding author
}

To cite this article:

Audrey Rae Norby, Caitlin Madden Clifford, Deborah Rose Berman. A Rare Arthrogryposis Syndrome with Multiple Anomalies Diagnosed by Whole Exome Sequencing. Journal of Gynecology and Obstetrics. Vol. 9, No. 4, 2021, pp. 128-131. doi: 10.11648/j.jgo.20210904.17

Received: May 24, 2021; Accepted: June 29, 2021; Published: August 31, 2021

\begin{abstract}
A 31-year-old gravida 1 para 0 was referred to our institution following a fetal anatomic survey demonstrating clubbed feet, flexed wrists, and skin edema. Ultrasound evaluation demonstrated these findings in addition to hemivertebrae, short long bones, contractures of the elbows, wrists, knees, and ankles with limited movement at the shoulders and hips. Further, macrocephaly, microphthalmia, low set ears, micrognathia, hepatomegaly, and an omphalocele were noted. Following termination of the pregnancy, whole exome sequencing ultimately identified compound heterozygous mutations in the NEK9 gene. One mutation in our case, c.136G>T, has never been reported; the other, c.1432del, has been reported once. To date, NEK9 mutations have been documented in three families with all affected individuals diagnosed with arthrogryposis. Our patient underwent targeted gene variant testing in two subsequent pregnancies, confirming identification of one of the two familial NEK9 gene mutations each time. Both pregnancies culminated in term deliveries of healthy neonates. This case illustrates a diagnosis of an extremely rare single gene disorder in the pregnancy of a non-consanguineous German couple, providing further evidence toward arthrogryposis with other anomalies as a recessive disease associated with NEK9 gene mutations. Finally, this case demonstrates whole exome sequencing as a valuable adjunct tool for investigating etiology when multiple fetal anomalies are identified without diagnosis on more standard tests.
\end{abstract}

Keywords: Arthrogryposis, Whole Exome Sequencing, NEK9

\section{Introduction}

Three to five percent of all pregnancies are complicated by fetal malformations or genetic syndromes [1]. Arthrogryposes are a clinically heterogeneous class of diseases with common features including congenital, usually non-progressive, joint contractures involving at least two different body areas [2]. Arthrogryposes affect approximately one in 3,000 live births and have varying etiologies including numerous genetic syndromes with differing inheritance patterns [3]. Unfortunately, traditional cytogenetic analyses, including karyotype and microarray, do not yield a diagnosis in the majority of arthrogryposis cases and oftentimes fail to rule out single gene disorders, which carry a $25 \%$ or higher risk of recurrence. Multi-gene panel testing is available for many conditions and anomaly phenotypes including arthrogryposis, but is not always practical given finite sample amounts, timelines, and associated costs. Without a specific diagnosis, counseling on anticipated outcomes including reproductive risks, options, and decision-making, may be limited [4]. Here we describe a case of a rare arthrogryposis syndrome caused by mutations in the NEK9 gene. This case illustrates an example of a single gene disorder in a previously undescribed population and demonstrates the value of whole exome sequencing as a prenatal diagnostic tool.

\section{Case Presentation}

A 31-year-old gravida 1 para 0 presented for a fetal anatomic survey at twenty weeks gestation. Ultrasound imaging revealed suspicion for clubbed feet, flexed wrists, and skin edema around the fetal abdomen. Providers obtained cell free fetal DNA, which revealed low risk for trisomies 13 , 18, and 21. Maternal serum AFP was elevated at 2.44 multiples of the median (MoM). The patient was 
subsequently referred to our institution at $23 \quad 1 / 7$ weeks gestation for further evaluation. Comprehensive ultrasound imaging demonstrated bilateral clubbed feet and flexed wrists. Fixed arm positions, short femurs, hemivertebrae, and trunk edema were also noted. There were contractures of the ankles, knees, elbows, and wrists with limited movement at the shoulders and hips (Figure 1). Hepatomegaly, omphalocele, macrocephaly, microphthalmia, low set ears, micrognathia, scalp and skin edema, and a small thorax were noted as well. Long bone measurements lagged by greater than two weeks.

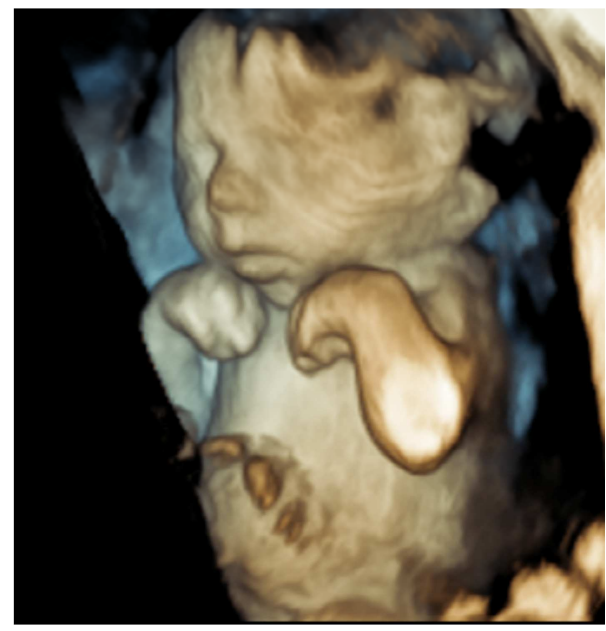

Figure 1. A three-dimensional antenatal ultrasound image demonstrating contractures of the ankles, knees, elbows, and wrists.

The couple met with maternal fetal medicine (MFM) as well as a prenatal genetic counselor. An unremarkable family pedigree was obtained. Counseling focused on the implications of the complex anomalies with involvement of multiple organ systems, possible etiologies with concern for an underlying genetic cause, and management options. The unusual constellation of findings raised concern for possible causes including skeletal dysplasia, arthrogryposis, or a metabolic disorder. After extensive counseling, the patient opted for termination of the pregnancy. Prior to termination, an amniocentesis was performed. Cultured amniocytes were sent for microarray as well as a lysosomal storage disease enzyme panel due to the hepatomegaly in the setting of this unusual constellation of findings.

Pathologic evaluation of the fetus demonstrated marked arthrogryposis of the upper and lower extremities with clubbing of the hands and feet and micromelia with short long bones, as demonstrated in postnatal imaging (Figure 2). There was bilateral clinodactyly and syndactyly, a short webbed neck with dysmorphic facial features including low set ears, microphthalmia, hypertelorism, synophrys, a long philtrum, depressed nasal bridge, and micrognathia. Short ribs with a small chest and small hypoplastic lungs were noted. A skin covered ventral wall defect, measuring $3.5 \mathrm{x}$ $1.8 \times 1.2 \mathrm{~cm}$, with liver herniation was present. The liver was histologically normal. Finally, subcutaneous edema was noted.

Microarray results revealed a variant of unknown significance: a $0.12 \mathrm{Mb}$ deletion of $2 \mathrm{q} 23.1$, which partially overlaps the MBD5 gene [5]. 2q23.1 deletion syndrome and $M B D 5$ variants are associated with neurodevelopmental abnormalities and subtle minor dysmorphic features but have not been reported in association with major structural anomalies as seen in this fetus $[5,6,7,8,9]$. Additionally, values were within normal limits for sixteen lysosomal enzymes. After further counseling, as the anomalies were not explained by this syndrome, whole exome sequencing was ordered on banked DNA from amniocytes. Two sequence variants were identified in NEK9 gene. The first, c.136G $>\mathrm{T}$ (maternal) was predicted to result in premature protein termination and had never before been reported. The second, c.1432del (paternal), was predicted to result in an amino acid frameshift and termination. This deletion had previously been observed in one out of 246,000 alleles in a large population database.

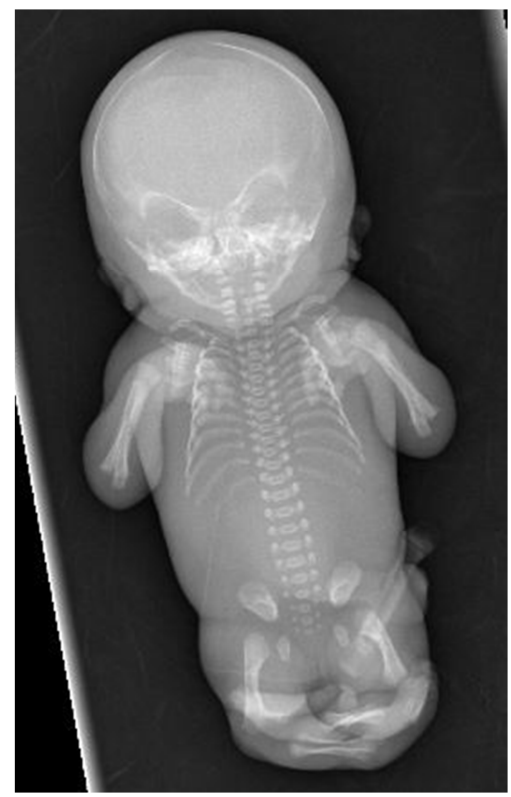

Figure 2. Postnatal imaging demonstrating marked arthrogryposis of the upper and lower extremities with clubbing of the hands and feet and micromelia with short long bones.

Following delivery, the patient met in consultation with genetic counseling and MFM. Exome sequencing results were discussed and implications for future pregnancies were reviewed. The patient was informed that while the laboratory classifies these variants as having uncertain significance, based on the clinical findings, we presumed that the compound heterozygous state was pathogenic and diseasecausing. Management options for future pregnancies included spontaneous conception with a likely $25 \%$ risk of recurrence, in vitro fertilization with transfer of an unaffected embryo, and alternative modes of parenting. The couple opted for spontaneous conception.

One year later, the patient presented to our Fetal Diagnostic Center for evaluation of viability and dating. A single live intrauterine pregnancy at eight weeks gestation was identified. After counseling, the patient opted to proceed with chorionic villus sampling (CVS). CVS was performed at 
$115 / 7$ weeks gestation and demonstrated a normal 46, XY karyotype. Targeted gene variant testing confirmed identification of one of the two familial NEK9 gene mutations. Fetal survey performed at 19 weeks gestation demonstrated normal anatomy. Follow up assessment demonstrated appropriate interval growth. The patient delivered a vigorous healthy liveborn male at 39 4/7 weeks of gestation. Two years later after conceiving again, she sought invasive targeted gene variant testing via CVS which again confirmed identification of one of the two familial NEK9 gene mutations. After an uncomplicated pregnancy, she delivered a healthy liveborn male.

\section{Discussion}

Following termination of the first pregnancy, whole exome sequencing identified compound heterozygous mutations in the NEK9 gene. NEK9 encodes a protein kinase that regulates cell cycle progression [10]. $N E K 9$ gain of function mutations have been implicated in pathogenesis of nevus comedonicus, a rare disorder involving inflammatory acne cysts, and are believed to disrupt normal follicular differentiation [11]. NEK9 has also been implicated in the progression of breast cancer and has been found to regulate spindle assembly and control chromosome alignment and centrosome separation [12]. NEK9 mutations have also thought to contribute to ciliary and cell cycle defects, with loss of function negatively impacting cell proliferation in multiple cases of lethal skeletal dysplasia [13]. Both were classified as variants of uncertain significance given the paucity of information on pathogenic NEK9 variants. One mutation in our case, c. $136 \mathrm{G}>\mathrm{T}$, has never been reported; the other, c.1432del, has been reported once. To date, NEK9 mutations are documented in three families: a consanguineous Saudi family homozygous for a missense variant c. $2042 \mathrm{G}>\mathrm{A} \quad[14,15]$, and two consanguineous Irish Traveler families with multiple affected individuals homozygous for a nonsense variant c. 1489C $>$ T [13]. All affected patients had arthrogryposis. Arthrogryposis, Perthes disease, subtle facial deformities, and an upward gaze palsy was noted in the Saudi patient homozygous for the c. $2042 \mathrm{G}>\mathrm{A}$ variant. The Irish Traveler pregnancy phenotypes closely mirrored our case with fetal akinesia, contractures, short long bones, rib anomalies, pulmonary hypoplasia, and protruding abdomens in multiple fetuses from the two families.

Arthrogryposis is a broad descriptive diagnosis for which it is challenging to ascertain etiology in a prenatal setting. Standard tests such as microarray often fail to yield a diagnosis in these cases. Homozygous variants in the NEK 9 gene have been associated with the phenotype of arthrogryposis with multiple other anomalies. Identification of these compound heterozygous NEK9 variants in this affected pregnancy raises suspicion of a causative relationship for an autosomal recessive syndrome, which would be associated with $25 \%$ recurrence risk. The subsequent pregnancy for this couple was identified to have one parental variant (c.136G $>\mathrm{T})$ and normal fetal anatomy ultrasound at 19 weeks gestation. This report highlights the importance of using whole exome sequencing for investigating the etiology of multiple fetal anomalies in cases without a causative diagnosis.

\section{Conclusion}

This case illustrates a diagnosis of an extremely rare single gene disorder in the pregnancy of a nonconsanguineous German couple. To our knowledge, this is the first case reported outside of Saudi and Irish Traveler populations. This provides further evidence toward arthrogryposis with other anomalies as a recessive disease associated with NEK9 gene mutations. Furthermore, this case demonstrates whole exome sequencing as a valuable adjunct tool for investigating etiology when multiple fetal anomalies are identified without diagnosis on more standard tests such as microarray. This information allowed comprehensive counseling for this couple regarding diagnosis, recurrence risk, and future reproductive options. Further, this permitted for targeted analysis at her subsequent pregnancies, providing reassuring information regarding an unaffected pregnancy.

\section{References}

[1] Dar PE, Shani H, Evans MI. Cell-free DNA: comparison of technologies. Clinics in Laboratory Medicine. 2016 Mar 18; 36 (2): 199-211.

[2] Kowalczyk B, Feluś J. Arthrogryposis: an update on clinical aspects, etiology, and treatment strategies. Archives of medical science: AMS. 2016 Feb 1; 12 (1): 10.

[3] Hall JG. Genetic aspects of arthrogryposis. Clinical orthopaedics and related research. 1985 Apr (194): 44-53.

[4] Filges I, Tercanli S, Hall JG. Fetal arthrogryposis: Challenges and perspectives for prenatal detection and management. In American Journal of Medical Genetics Part C: Seminars in Medical Genetics 2019 Sep (Vol. 181, No. 3, pp. 327-336). Hoboken, USA: John Wiley \& Sons, Inc..

[5] Mullegama SV, Elsea SH. Clinical and molecular aspects of MBD5-associated neurodevelopmental disorder (MAND). European Journal of Human Genetics. 2016 Sep; 24 (9): 123543.

[6] Jaillard S, Dubourg C, Gérard-Blanluet M, Delahaye A, Pasquier L, Dupont C, Henry C, Tabet AC, Lucas J, Aboura A, David V. 2q23. 1 microdeletion identified by array comparative genomic hybridisation: an emerging phenotype with Angelman-like features?. Journal of medical genetics. 2009 Dec 1; 46 (12): 847-55.

[7] Talkowski ME, Mullegama SV, Rosenfeld JA, Van Bon BW, Shen Y, Repnikova EA, Gastier-Foster J, Thrush DL, Kathiresan S, Ruderfer DM, Chiang C. Assessment of 2q23. 1 microdeletion syndrome implicates MBD5 as a single causal locus of intellectual disability, epilepsy, and autism spectrum disorder. The American Journal of human genetics. 2011 Oct 7; 89 (4): 551-63. 
[8] Noh GJ, Graham Jr JM. 2q23. 1 microdeletion of the MBD5 gene in a female with seizures, developmental delay and distinct dysmorphic features. European journal of medical genetics. 2012 May 1; 55 (5): 354-7.

[9] Van Bon BW, Koolen DA, Brueton L, McMullan D, Lichtenbelt KD, Adès LC, Peters G, Gibson K, Novara F, Pramparo T, Dalla Bernardina B. The 2q23. 1 microdeletion syndrome: clinical and behavioural phenotype. European journal of human genetics. $2010 \mathrm{Feb} ; 18$ (2): 163-70.

[10] O'regan L, Blot J, Fry AM. Mitotic regulation by NIMArelated kinases. Cell division. 2007 Dec; 2 (1): 1-2.

[11] Levinsohn JL, Sugarman JL, McNiff JM, Antaya RJ, Choate KA. Somatic mutations in NEK9 cause nevus comedonicus. The American Journal of Human Genetics. 2016 May 5; 98 (5): 1030-7.

[12] Xu Z, Shen W, Pan A, Sun F, Zhang J, Gao P, Li L. Decreased Nek9 expression correlates with aggressive behaviour and predicts unfavourable prognosis in breast cancer. Pathology. 2020 Apr 1; 52 (3): 329-35.

[13] Casey JP, Brennan K, Scheidel N, McGettigan P, Lavin PT, Carter S, Ennis S, Dorkins H, Ghali N, Blacque OE, Mc Gee MM. Recessive NEK9 mutation causes a lethal skeletal dysplasia with evidence of cell cycle and ciliary defects. Human molecular genetics. 2016 May 1; 25 (9): 1824-35.

[14] Alkuraya FS. Arthrogryposis, perthes disease, and upward gaze palsy: a novel autosomal recessive syndromic form of arthrogryposis. American Journal of Medical Genetics Part A. $2011 \mathrm{Feb} ; 155$ (2): 297-300.

[15] Shaheen R, Patel N, Shamseldin H, Alzahrani F, Al-Yamany R, ALMoisheer A, Ewida N, Anazi S, Alnemer M, Elsheikh M, Alfaleh K. Accelerating matchmaking of novel dysmorphology syndromes through clinical and genomic characterization of a large cohort. Genetics in Medicine. 2016 Jul; 18 (7): 686-95. 